\title{
Ranking fuzzy numbers using fuzzy maximizing-minimizing points
}

\author{
S. Salahshour*1 S. Abbasbandy ${ }^{2}$ T. Allahviranloo ${ }^{2}$ \\ ${ }^{1}$ Department of Mathematics, Mobarakeh Branch, Islamic Azad University, Mobarakeh, Iran. \\ ${ }^{2}$ Department of Mathematics, Science and Research Branch, Islamic Azad University, Tehran, Iran.
}

\begin{abstract}
Up to now, several approaches have been proposed for ranking of fuzzy numbers using maximizing set and minimizing sets. Often, the reference points are created by real numbers. In this paper, we proposed new reference points so called fuzzy maximizing-minimizing points. To this purpose, we used the center of gravity of fuzzy numbers and their left/right spreads to construct such fuzzy points. Moreover, we suggested a new aggregation function with pessimistic/optimistic structure for a set of fuzzy numbers. Also, some examples are given to show the ability of proposed ranking approach and the related aggregation function.
\end{abstract}

Keywords: Fuzzy numbers, Fuzzy maximizingminimizing points, Ranking, Center of gravity (COG) point, Aggregation.

\section{Introduction}

Fuzzy set theory [20] has been applied to many areas which need to manage uncertain and vague data. Such areas include approximate reasoning, decision making, optimization, control and so on, where ranking of fuzzy numbers is an important component of the decision process $[19,21]$.

A key issue in the fuzzy set theory is how to compare fuzzy numbers. Several methods have been developed for raking fuzzy numbers $[1,4,17,18]$. Some of these ranking approaches have compared and reviewed by Bortolan and Degani [5]. Recently, Chen and Hwang [7] reviewed the existing methods for ranking fuzzy numbers. Moreover, Wang and Kerre [12] proposed some axioms as reasonable properties to determine the rationality of a fuzzy number ranking approach and systematically compared a wide array of the existing fuzzy number ranking methods. Almost each approach, however, has pitfalls in some aspect, such as inconsistency with human intuition, indiscrimination, and difficulty of interpretation. So far, none of them is commonly and completely accepted.

*Corresponding author: Tell:+989155154014. Email address: soheilsalahshour@yahoo.com (S. Salahshour)
Chen [6] proposed the maximizing set and minimizing set method. Recently, Wang and Luo [15], suggested an alternative ranking approach for fuzzy numbers so-called area ranking based on positive and negative ideal points. Also, Wang et. al [14] proposed a novel approach to ranking fuzzy numbers based on the left and right deviation degree. To this end, the maximal and minimal reference sets are defined to measure the deviation degree of fuzzy numbers. Please note that, in all of the existing ranking approaches based on the maximizing and minimizing reference sets, these reference sets are chosen as crisp (real) numbers.

We believe that " it is reasonable that the maximizing point and minimizing point for a set of fuzzy numbers should be stated by fuzzy numbers instead of crisp number". So, we propose the fuzzy maximizing point and the fuzz minimizing point. To do this purpose, we decided to use the COG points and the left/right spreads of fuzzy numbers. Note that, constructing of an unknown fuzzy number by its COG point and its left/right spreads was introduced by Hadi et. al [10]. Also, our proposed method has one another significant property such that we can aggregate a set of fuzzy numbers under the pessimistic/optimistic manners.

The paper is organized as follows. Section 2 briefly reviews the basic concepts. In Section 3, the COG point of fuzzy number is stated briefly and then the Hadi et. al's method [10] is reviewed. In Section 4, we propose new ranking approach using COG points and left-right spreads. Moreover, a new pessimistic/optimistic aggregation function is proposed. For convenience, an algorithm is expressed to fine out the proposed method better. In Section 5 , Several examples are solved and compared with the previously reported ranking approaches. The paper ends with a short conclusion.

\section{Basic concepts}

In this section, some basic concepts which will be used later are given.

The most commonly used fuzzy numbers are triangular and trapezoidal fuzzy numbers, whose 
membership functions are respectively defined as

$$
\begin{gathered}
\mu_{A}(x)= \begin{cases}\left(\frac{x-a_{1}}{a_{2}-a_{1}}\right), & a_{1} \leq x \leq a_{2}, \\
\left(\frac{a_{3}-x}{a_{3}-a_{2}}\right), & a_{2} \leq x \leq a_{3}, \\
0, & \text { Otherwise, }\end{cases} \\
\mu_{B}(x)= \begin{cases}\left(\frac{x-a_{1}}{a_{2}-a_{1}}\right), & a_{1} \leq x \leq a_{2}, \\
1, & a_{2} \leq x \leq a_{3}, \\
\left(\frac{a_{4}-x}{a_{4}-a_{3}}\right), & a_{3} \leq x \leq a_{4}, \\
0, & \text { Otherwise, }\end{cases}
\end{gathered}
$$

For brevity, triangular and trapezoidal fuzzy numbers are often denoted as $A=\left(a_{1}, a_{2}, a_{3}\right)$ and $B=\left(a_{1}, a_{2}, a_{3}, a_{4}\right)$. Clearly, triangular fuzzy numbers are special cases of trapezoidal fuzzy numbers with $a_{2}=a_{3}$.

An equivalent parametric definition is also given in [8] as follows:

Definition 1 A fuzzy number $u$ in parametric form is a pair $(\underline{u}, \bar{u})$ of functions $\underline{u}(r), \bar{u}(r), 0 \leq r \leq 1$, which satisfy the following requirements:

1. $\underline{u}(r)$ is a bounded non-decreasing left continuous function in $(0,1]$, and right continuous at 0 ,

2. $\bar{u}(r)$ is a bounded non-increasing left continuous function in $(0,1]$, and right continuous at 0 ,

3. $\underline{u}(r) \leq \bar{u}(r), 0 \leq r \leq 1$.

According to Zadeh's extension principle, the operation of addition on $\mathbb{E}$ is defined by

$$
(u+v)(x)=\sup _{y \in \mathbb{R}} \min \{u(y), v(x-y)\}, \quad x \in \mathbb{R},
$$

and scalar multiplication of a fuzzy number is given by

$$
(k \odot u)(x)=\left\{\begin{array}{l}
u(x / k), \quad k>0, \\
\widetilde{0}, \quad k=0,
\end{array}\right.
$$

where $\tilde{0} \in \mathbb{E}$.

Definition 2 For arbitrary fuzzy numbers $A$ and $B$, the quantity

$d(A, B)=\left[\int_{0}^{1}(\underline{A}(r)-\underline{B}(r))^{2} d r+\int_{0}^{1}(\bar{A}(r)-\bar{B}(r))^{2} d r\right]^{\frac{1}{2}}$ is the distance between $A$ and $B$. For more detail see [9].

\section{Center of gravity (COG) point of fuzzy number}

Let us consider $A$ is a given fuzzy number such that $A=\left(a_{1}, a_{2}, a_{3}, a_{4}\right)$, then the centroid point of $A$ is obtained by Wang et. al [16] by

$$
\operatorname{COG}(A)=\left(\bar{x}_{0}(A), \bar{y}_{0}(A)\right),
$$

where

$$
\left\{\begin{array}{l}
\bar{x}_{0}(A)=\frac{1}{3}\left[a_{1}+a_{2}+a_{3}+a_{4}-\frac{a_{4} a_{3}-a_{1} a_{2}}{\left(a_{4}+a_{3}\right)-\left(a_{1}+a_{2}\right)}\right], \\
\bar{y}_{0}(A)=\frac{1}{3}\left[1+\frac{a_{3}-a_{2}}{\left(a_{4}+a_{3}\right)-\left(a_{1}+a_{2}\right)}\right] .
\end{array}\right.
$$

Hence, for any triangular fuzzy number with a piecewise linear membership function, its centroid can be derived by

$$
\begin{aligned}
& \bar{x}_{0}(A)=\frac{a_{1}+a_{2}+a_{4}}{3}, \\
& \bar{y}_{0}(A)=\frac{1}{3} .
\end{aligned}
$$

Moreover, we need the method which was proposed by Hadi et.al [10] for determining the fuzzy maximizing-minimizing points according to the center of gravity points of fuzzy numbers and their left/right spreads. So, for convenience, a briefly review of this method is given:

Let us consider the left and right spreads, $(L, R)$, and COG point $\left(\bar{x}_{0}(A), \bar{y}_{0}(A)\right)$ of unknown fuzzy number $A$ are given. The goal is determining such unknown fuzzy number uniquely. To do this purpose, we should solve the following linear system:

$$
\left\{\begin{array}{l}
a_{2}-a_{1}=L \\
a_{4}-a_{3}=R \\
\bar{x}_{0}(A)=\frac{1}{3}\left[a_{1}+a_{2}+a_{3}+a_{4}-\frac{a_{4} a_{3}-a_{1} a_{2}}{\left(a_{4}+a_{3}\right)-\left(a_{1}+a_{2}\right)}\right] \\
\bar{y}_{0}(A)=\frac{1}{3}\left[1+\frac{a_{3}-a_{2}}{\left(a_{4}+a_{3}\right)-\left(a_{1}+a_{2}\right)}\right] .
\end{array}\right.
$$

Note that, for more details see [10].

\section{The proposed method}

In this section, we propose a new approach for ranking fuzzy numbers using fuzzy maximizingminimizing points which are constructed by their center of gravity points. To do this purpose, let us consider $n$ fuzzy numbers $A_{i}, i=1,2, \ldots, n$, are given. Then, in order to construct the fuzzy maximizing-minimizing points which are denoted by $M$ and $m$, respectively, we adopted the following procedure:

Let us consider the COG point of $A_{i}, i=1,2, \ldots, n$ are denoted by:

$\operatorname{COG}\left(A_{i}\right)=\left(\bar{x}_{0}\left(A_{i}\right), \bar{y}_{0}\left(A_{i}\right)\right), \quad i=1,2, \ldots, n$.

Then, using Hadi's approach [10], we can obtain an unique fuzzy number with its COG point and left right spreads. So, we produce the fuzzy maximizing-minimizing points using COG points and left-right spreads of given fuzzy numbers $A_{i}$ as follows:

The COG point of fuzzy maximizing point $(M)$ and fuzzy minimizing point $m$ are derived by

$C O G(M)=\left(\max _{i=1,2, \ldots, n}\left\{\bar{x}_{0}\left(A_{i}\right)\right\}, \max _{i=1,2, \ldots, n}\left\{\bar{y}_{0}\left(A_{i}\right)\right\}\right)$ 
and

$C O G(m)=\left(\min _{i=1,2, \ldots, n}\left\{\bar{x}_{0}\left(A_{i}\right)\right\}, \min _{i=1,2, \ldots, n}\left\{\bar{y}_{0}\left(A_{i}\right)\right\}\right)$

Also, we chose the following procedure for determining the left-right spreads of fuzzy maximizing point:

$$
\begin{aligned}
L_{M} & =\max _{i=1,2, \ldots, n}\left\{L_{A_{i}}\right\} \\
R_{M} & =\max _{i=1,2, \ldots, n}\left\{R_{A_{i}}\right\},
\end{aligned}
$$

where $L_{M}$ and $R_{M}$ are the left and the right spreads of fuzzy maximizing point $M$, respectively. Also, $L_{A_{i}}$ and $R_{A_{i}}$ are the left and the right spreads of given fuzzy numbers $A_{i}, i=1,2, \ldots, n$. Similarly, we construct the left and the right spreads of fuzzy minimizing point as follows:

$$
L_{m}=\min _{i=1,2, \ldots, n}\left\{L_{A_{i}}\right\}
$$

and

$$
R_{m}=\min _{i=1,2, \ldots, n}\left\{R_{A_{i}}\right\} .
$$

Now, the fuzzy maximizing-minimizing points could be constructed using their COG points and left-right spreads using Hadi's approach [10].

Up to now, we built the fuzzy maximizingminimizing points. But, the other essential part is selecting a common way for ranking fuzzy numbers using these fuzzy points. To do this purpose, we introduce the following relative closeness coefficient of a fuzzy number with respect to the fuzzy maximizing-minimizing points given by:

$$
D(A)=\gamma(A) \times \frac{D_{A}^{L}}{1+D_{A}^{R}}
$$

where

$$
\begin{aligned}
& D_{A}^{L}=d(A, m), \\
& D_{A}^{R}=d(A, M),
\end{aligned}
$$

and

$$
\gamma(A)=\left\{\begin{array}{l}
1, \quad \text { if } \quad \int_{0}^{1}\{\underline{A}(r)+\bar{A}(r)\} d r \geq 0 \\
-1, \quad \text { if } \quad \int_{0}^{1}\{\underline{A}(r)+\bar{A}(r)\} d r<0
\end{array}\right.
$$

Finally, the ranking of $n$ fuzzy numbers $A_{1}, A_{2}, \ldots, A_{n}$ is determined by the following rules for all $i, j=1,2, \ldots, n$ :

(1) $A_{i} \prec A_{j}$ if and only if $D\left(A_{i}\right)<D\left(A_{j}\right)$,

(2) $A_{i} \succ A_{j}$ if and only if $D\left(A_{i}\right)>D\left(A_{j}\right)$,

(3) $A_{i} \approx A_{j}$ if and only if $D\left(A_{i}\right)=D\left(A_{j}\right)$.

Consequently, the Wang's and Kerre's reasonable properties are investigated for the ordering approach (see[12]).
A-1 For an arbitrary finite subset $\mathcal{A}$ of $S$ and $u \in \mathcal{A} ; u \succeq u$.

(4) A-2 For an arbitrary finite subset $\mathcal{A}$ of $S$ and $(u, v) \in \mathcal{A}^{2} ; \quad u \succeq v$ and $v \succeq u$ by $D$ on $\mathcal{A}$, this method should have $u \sim v$.

A-3 For an arbitrary finite subset $\mathcal{A}$ of $S$ and $(u, v, w) \in \mathcal{A}^{3} ; \quad u \succeq v$ and $v \succeq w$ by $D$ on $\mathcal{A}$, this method should have $u \succeq w$.

A-4 For an arbitrary finite subset $\mathcal{A}$ of $S$ and $(u, v) \in \mathcal{A}^{2} ; \quad \inf \operatorname{supp}(u)>\sup \operatorname{supp}(v)$, this method should have $u \succeq v$.

$A^{\prime}-4$ For an arbitrary finite subset $\mathcal{A}$ of $S$ and $(u, v) \in \mathcal{A}^{2} ; \quad \inf \operatorname{supp}(u)>\sup \operatorname{supp}(v)$, this method should have $u \succ v$.

A-5 Let $S, S^{\prime}$ be two arbitrary finite sets of fuzzy quantities in which $D$ can be applied and $u, v$ are in $S \cap S^{\prime}$. This method obtain the ranking order $u \succeq v$ on $S^{\prime}$ iff $u \succeq v$ on $S$.

A-6 Let $u, v, u+w$ and $v+w$ be elements of $S$. If $u \succeq v$ by $D$ on $u, v$, then $u+w \succeq v+w$.

$A^{\prime}-6$ Let $u, v, u+w$ and $v+w$ be elements of $S$. If $u \succ v$ by $D$ on $u, v$, then $u+w \succ v+w$.

Proposition 1 The proposed distance $D$ has the above properties.

\subsection{Aggregation of fuzzy numbers}

Let us consider a decision group has $P$ decision makers and the fuzzy rating of each decision maker $D_{p},(p=1,2, \ldots, P)$, can be expressed as fuzzy numbers $R_{p}$. An useful aggregation method must be considered the range of fuzzy rating of each decision maker. Recently, some aggregation methods were proposed. Guneri et.al [11] proposed a method based on the maximum and minimum values of the end points of supports of given fuzzy numbers. Consequently, Hadi et. al [10] proposed a new way for aggregating several fuzzy numbers using their COG points and left-right spreads.

We introduce another new way to aggregate given fuzzy numbers to a fuzzy number using COG points. In accordance with the our proposed method in the previous section, two obtained fuzzy numbers $M$ and $m$ can be considered as some aggregation of given fuzzy numbers. In order to improve such aggregation, we proposed a pessimistic/optimistic approach as follows:

$$
\operatorname{Agg}(\Gamma ; \lambda)=\lambda \odot M+(1-\lambda) \odot m,
$$

for all $\lambda$ in $[0,1]$. Note that $\Gamma$ denotes a set of $n$ fuzzy numbers $\left\{A_{1}, A_{2}, \ldots, A_{n}\right\}$. Clearly, for $\lambda=0$, the aggregation function is equal to $m$ which is the pessimistic case and for $\lambda=1$, the aggregation function is equal to $M$ which is the optimistic case.

Now, we summarize our discussions as follows. Using Eqs. (3-4), we can construct the centriod points of given fuzzy numbers both triangular 
and trapezoidal fuzzy numbers. Since, in our opinion, the maximizing point should possess the biggest parameters in each cases. For example, should possess the biggest left/right spreads and the biggest COG point. Obviously, this is valid for the minimizing point which should possess the smallest parameters. Simultaneously, we derive the left/right spreads for such reference points using Eqs. (5-6) and Eqs. (7-8). Clearly, these selections coincide with our procedure. Then, using interesting method which was proposed by Hadi et. al [10], the unknown reference points are constructed. In addition, we proposed a new ranking method. Using it, we can rank given fuzzy numbers in compare of obtained maximizing-minimizing points. Again, proposed ordering approach coincides with our thinks. As a novel application, we determine the pessimistic/optimistic aggregation function by applying linear combination of the maximizing and minimizing points.

At the end of this part, we present an algorithm in order to state the method better as follows:

\section{Algorithm:}

Let us consider $n$ fuzzy numbers $A_{1}, A_{2}, \ldots, A_{n}$ are given. Then,

- The COG points of fuzzy maximizing-minimizing points are derived by Eqs. (3-4).

- The left and the right spreads of fuzzy maximizing and minimizing points are obtained using Eqs. (5-6) and Eqs. (7-8).

- The unknown fuzzy maximizing-minimizing points will be determined using Hadi's method [10].

- The ordering of fuzzy numbers will be done using Eqs. (9-12).

- The pessimistic/optimistic aggregation function is denoted by $\operatorname{Agg}(\Gamma ; \lambda)$ which is evaluated by Eq. (13).

\section{Examples}

In this section, we take several examples in order to show the ability of proposed method in compare of recently published ordering approaches.

Example 1 Let us consider two fuzzy numbers $A=(1,2,6)$ and $B=(2,3,4)$, then using our proposed method, the fuzzy maximizing point $M$ and the fuzzy minimizing point $m$ are determined as fol- lows:

$$
\begin{aligned}
& \operatorname{COG}(M)=\left(3, \frac{1}{3}\right), \quad L_{M}=1, R_{M}=4 \\
& \operatorname{COG}(m)=\left(3, \frac{1}{3}\right), \quad L_{m}=1, R_{m}=1
\end{aligned}
$$

then, using Hadi's approach [10], these fuzzy points are obtained as follows:

$$
M=(1,2,6) \quad \& \quad m=(2,3,4) .
$$

Now, using the proposed relative closeness coefficient of a fuzzy number with respect to the fuzzy maximizing-minimizing points, we have:

$$
\begin{aligned}
& D_{A}^{L}=d(A, m)=\sqrt{2}, \\
& D_{A}^{R}=d(A, M)=0,
\end{aligned}
$$

and, also

$$
\begin{aligned}
& D_{B}^{L}=d(B, m)=0, \\
& D_{B}^{R}=d(B, M)=\sqrt{2} .
\end{aligned}
$$

Therefore, we get:

$$
D(A)=\sqrt{2}>D(B)=0 .
$$

Hence, in accordance with the proposed ordering, we get: $A \succ B$ which agrees with the previous result [3]. However, we can logically infer that the ordering of images of these fuzzy numbers is as $-A \prec-B$. Clearly, using our proposed method, the fuzzy maximizing-minimizing points for images of given fuzzy number $A, B$ are derived:

$$
M=(-6,-2,-1), \quad \& \quad m=(-4,-3,-2),
$$

and then, we get:

$$
D(-A)=-\sqrt{2}, \quad D(-B)=0 .
$$

Thus, the ranking of images of given fuzzy numbers is $-A \prec-B$. Obtained results agree with Asady's result [3] and is the shortcoming of proposed method by Wang et.al [14]. Since, by using Wang's method [14], the unreasonable ordering $-A \approx-B$ is derived.

Remark 1 By assumptions of Example 1, we can aggregate given set of fuzzy numbers $\Gamma=\{A, B\}$ by

$$
\begin{aligned}
\operatorname{Agg}(\Gamma ; \lambda) & =\lambda \odot M+(1-\lambda) \odot m \\
& =\lambda \odot(1,2,6)+(1-\lambda) \odot(2,3,4) \\
& =(2-\lambda, 3-\lambda, 4+2 \lambda),
\end{aligned}
$$

for all $\lambda$ in $[0,1]$. Also, using Hadi's approach [10], the aggregated fuzzy rating $R_{H}$ is obtained as follows:

$$
R_{H}=\left(\frac{3}{2}, \frac{5}{2}, \frac{10}{2}\right)
$$


which agrees with our proposed aggregated fuzzy rating $\operatorname{Agg}(\Gamma ; \lambda)$ by considering intermediate case $\lambda=$ $\frac{1}{2}$. It is easy to verify that our sensitive aggregated method is an extension of Hadi's approach [10]. In addition, using Guneri's method [11] we have:

$$
R_{G}=\left(1, \frac{5}{2}, 6\right)
$$

So, we have the following relation between obtained aggregated fuzzy numbers:

$$
\begin{aligned}
\operatorname{Agg}\left(\Gamma ; \frac{1}{2}\right) & =R_{H} \\
& \subset R_{G} .
\end{aligned}
$$

Example 2 Let us consider three fuzzy numbers:

$$
\begin{aligned}
& A=(5,6,7), \\
& B=(5.9,6,7), \\
& C=(6,6,7) .
\end{aligned}
$$

Then, we get:

$$
\begin{aligned}
& C O G(M)=\left(\frac{19}{3}, \frac{1}{3}\right), \quad L_{M}=1, R_{M}=1 \\
& \operatorname{COG}(m)=\left(6, \frac{1}{3}\right), \quad L_{m}=0, R_{m}=1
\end{aligned}
$$

Therefore, by using our proposed method, the fuzzy maximizing-minimizing points are determined as follows:

$m=\left(\frac{17}{3}, \frac{17}{3}, \frac{20}{3}\right) \quad \& \quad M=\left(\frac{16}{3}, \frac{19}{3}, \frac{22}{3}\right)$,

and then, using simple calculations, we get:

$$
D(A)<D(B)<D(C) .
$$

Consequently, the ranking is $A \prec B \prec C$ which agrees with the Abbasbandy et.al's methods [1, 2] and Wang et.al approach [14].

Remark 2 In order to construct a pessimistic/optimistic aggregation using Example 2, we have:

$\operatorname{Agg}(\Gamma ; \lambda)=\lambda \odot\left(\frac{17}{3}, \frac{17}{3}, \frac{20}{3}\right)+(1-\lambda) \odot\left(\frac{16}{3}, \frac{19}{3}, \frac{22}{3}\right)$,

where $\Gamma=\{A, B, C\}$. Note that, the aggregated fuzzy numbers using Hadi's approach [10] and Guneri's approach [11] is obtained as follows:

$$
R_{H}=(5.636,6,7) \quad \& \quad R_{G}=(5,6,7) .
$$

Also, for intermediate case $\frac{1}{2}$, we have:

$$
\begin{aligned}
R_{H} \subset \operatorname{Agg}\left(\Gamma ; \frac{1}{2}\right) & =\left(\frac{11}{2}, 6,7\right) \\
& \subset R_{G} .
\end{aligned}
$$

Example 3 Let us consider two fuzzy numbers $A=(3,6,9)$ and $B=(5,6,7)$. Then, the $C O G$ points and left-right spreads of fuzzy maximizingminimizing points are obtained as follows:

$$
\begin{aligned}
& C O G(M)=\left(6, \frac{1}{3}\right), L_{M}=3, R_{M}=3 \\
& C O G(m)=\left(6, \frac{1}{3}\right), \quad L_{m}=1, R_{m}=1 .
\end{aligned}
$$

Consequently, the reference points $M$ and $m$ are determined as follows:

$$
M=(3,6,9) \quad \& \quad m=(5,6,7) .
$$

Then, we get:

$$
D(A)=d(A, m)>0, \quad \& \quad D(B)=0,
$$

therefore, the ordering is derived as $A \succ B$ which agrees with the previously reported result [14]. However, using Abbasbandy's results [1, 2], the ordering is derived $A \approx B$, which is not intuitionally correct.

Remark 3 Using assumptions of Example 3, we have below aggregation:

$$
\begin{aligned}
\operatorname{Agg}(\Gamma ; \lambda) & =\lambda \odot(3,6,9)+(1-\lambda) \odot(5,6,7) \\
& =(5-2 \lambda, 6,7+2 \lambda)
\end{aligned}
$$

where $\Gamma=\{A, B\}$. Note that the aggregated fuzzy numbers using Hadi's result [10] and Guneri's result [11] are obtained as follows:

$$
R_{H}=(4,6,8), \quad \& \quad R_{G}=(3,6,9) .
$$

It is easy to verify that we have the following relation in the intermediate case $\lambda=\frac{1}{2}$ :

$$
\begin{aligned}
\operatorname{Agg}\left(\Gamma ; \frac{1}{2}\right) & =R_{H} \\
& \subset R_{G} .
\end{aligned}
$$

Now, for emphasizing on the aggregate function, we take another example which has been taken from [13] and considered under new method by Hadi et.al [10].

Example 4 Let us consider five given fuzzy numbers:

$$
\begin{aligned}
& A_{1}=(8,9,10), \\
& A_{2}=(9,10,10), \\
& A_{3}=(7,8,9), \\
& A_{4}=(5,6,7), \\
& A_{5}=(6,7,8) .
\end{aligned}
$$

Then, the COG points of maximizing and minimizing points are obtained as follows:

$$
\begin{aligned}
C O G(M) & =\left(\frac{29}{3}, \frac{1}{3}\right), \quad L_{M}=1, R_{M}=1 \\
C O G(m) & =\left(6, \frac{1}{3}\right), \quad L_{m}=1, R_{m}=0
\end{aligned}
$$


Therefore, by using our proposed method, the fuzzy maximizing-minimizing points are determined as follows:

$M=\left(\frac{26}{3}, \frac{29}{3}, \frac{32}{3}\right) \quad \& \quad m=\left(\frac{16}{3}, \frac{19}{3}, \frac{19}{3}\right)$.

Then, using proposed aggregate function we have:

$\operatorname{Agg}(\Gamma ; \lambda)=\lambda \odot\left(\frac{26}{3}, \frac{29}{3}, \frac{32}{3}\right)+(1-\lambda) \odot\left(\frac{16}{3}, \frac{19}{3}, \frac{19}{3}\right)$,

where $\Gamma=\left\{A_{i}\right\}_{i=1}^{5}$. Simply, we get:

$\operatorname{Agg}(\Gamma ; \lambda)=\left(\frac{16+10 \lambda}{3}, \frac{19+10 \lambda}{3}, \frac{19+13 \lambda}{3}\right)$.

In addition, Hadi et.al [10] obtained the following aggregated result:

$$
R_{H}=\left(7,8, \frac{44}{5}\right)
$$

and using Guneri's method, we have:

$$
R_{G}=(5,8,10)
$$

Note that, for the intermediate case $\lambda=\frac{1}{2}$ we have the following result:

$$
\begin{aligned}
\operatorname{Agg}\left(\Gamma ; \frac{1}{2}\right) & =\left(7,8, \frac{17}{2}\right) \\
& \subset R_{H} \\
& \subset R_{G} .
\end{aligned}
$$

\section{Conclusion}

In this paper we have developed a new approach for ranking of fuzzy numbers. This approach considers not only the left and right spreads of fuzzy numbers, but also their COG points have been associated in order to construct the fuzzy maximizingminimizing points. Then, a new relative closeness coefficient with respect to such reference points are introduced which is the basis of our proposed ordering. Note that, our proposed method can be seen as a novel method for aggregate a set of fuzzy numbers to a fuzzy number. To this end, a new pessimistic/optimistic aggregate function is proposed. It is evident that proposed approach can be used for trapezoidal fuzzy numbers, since the proposed formula is general. Notice that, one can easily obtain a new aggregation function using another approach for constructing the fuzzy maximizing point and minimizing points. For future research, we will adopt the mentioned aggregated method for solving fuzzy MCDM problems.

\section{References}

[1] S. Abbasbandy and B. Asady, Ranking of fuzzy numbers by sign distance, Information Sciences 176 (2006) 2405-2416.
[2] S. Abbasbandy and T. Hajjari, A new approach for ranking of trapezoidal fuzzy numbers, Computer and Mathematics with Applications 57 (2009) 413-419.

[3] B. Asady, The revised method of ranking LR fuzzy numbers based on deviation degree, Expert Systems with Applications 37 (2010) 50565060.

[4] B. Asady and A. Zendehnam, Ranking fuzzy numbers by distance minimization, Applied Mathematical Modelling 31 (2007) 2589-2598.

[5] G. Bortolan and R. Degani, A review of some methods for ranking fuzzy subsets, Fuzzy Sets and Systems 15 (1985) 1-19.

[6] S.H. Chen, Ranking fuzzy numbers with maximizing set and minimizing set, Fuzzy Sets and Systems 17 (1985) 113-129.

[7] S.J. Chen and C.L. Hwang, Fuzzy Multiple Attribute Decision Making, Springer, Berlin, 1992.

[8] M. Friedman, M. Ma and A. Kandel, Numerical solution of fuzzy differential and integral equations, Fuzzy Sets and System 106 (1999) 35-48.

[9] P. Grzegorzewski, Nearest interval approximation of a fuzzy number, Fuzzy Sets and System 130 (2002) 321-330.

[10] A. Hadi-vencheh and M. Allame, On the relation between a fuzzy number and its centroid, Computer and Mathematics with Applications 59 (2010) 3578-3582.

[11] A.F. Guneri, A. Yucel and G. Ayyildiz, An integrated fuzzy-lp approach for a supplier selection problem in supply chain managment, Expert Systems with Applications 36 (2009) 92239228.

[12] X. Wang and E.E. Kerre, Reasonable properties for the ordering of fuzzy quantities, Fuzzy Sets and Systems 118 (2001) 375-385.

[13] Y.M. Wang, K.S. Chin, G.K.K. Poon and J.B. yang, Risk evaluation in failure mode and effects analysis using fuzzy weighted geometric mean, Expert Systems with Applications 36 (2009)1195-1207.

[14] Z.X. Wang, Y.J. Liu, Z.P. Fan and B. Feng, Ranking L-R fuzzy numbers based on deviation degree, Information Sciences 179 (2009) 20702077.

[15] Y.M. Wang and Y. Luo, Area ranking of fuzzy numbers based on positive and negative ideal points, Computers and Mathematics with Applications 58 (2009) 1769-1779.

[16] Y.M. Wanga, J.B. Yanga, D.L. Xua and K.S. Chinc, On the centroids of fuzzy numbers, Fuzzy Sets and Systems 157 (2006) 919-926.

[17] J.S. Yao and F.T. Lin, Fuzzy critical path method based on signed distance ranking of fuzzy numbers, IEEE Transactions on Systems Man, and Cybernetics, Part A: Systems and Humans 30 (2000) 76-82. 
[18] J.S. Yao and K. Wu, Ranking fuzzy numbers based on decomposition principle and signed distance, Fuzzy Sets and Systems 116 (2000) 275-288.

[19] A. Kauffman and M.M. Gupta, Introduction to Fuzzy Arithmetic: Theory and Application, Van Nostrand Reinhold, New York, 1991.

[20] L.A. Zadeh, Fuzzy Sets, Information and Control 8 (1965) 338-353.

[21] H.J. Zimmermann, Fuzzy sets theory and its applications, Kluwer Academic Press, Dordrecht, 1991. 